\title{
REDUCING UNCERTAINTY IN EXTREME WAVES AND STORM SURGES USING A COMBINED EXTREME VALUE MODEL AND WAVELETS
}

\author{
P. Galiatsatou ${ }^{1}$ and P. Prinos
}

\begin{abstract}
In the present study the wavelet transform is combined with non-stationary statistical models for extreme value analysis, to provide more reliable and more accurate return level estimates. The continuous wavelet transform is first used to detect the significant "periodicities" of the wave height and storm surge signals under study by means of the wavelet global and scale-averaged power spectra and then it is used to reconstruct the part of the time series, represented by these significant and prominent features. A non-stationary point process is utilized to model the extremes. A time varying threshold with a period of one year and having an approximately uniform crossing rate throughout the year is used. The reconstructed part of the series variability representing the significant nonstationarities of each signal is incorporated in the both the location and the scale parameters of the point process model, together with selected harmonic functions, formulating a number of candidate extreme value models. The quality of the fitted models is assessed by means of the Akaike Information Criterion, as well as by means of diagnostic quantile plots. The models which incorporate the reconstructed part of the wavelet transform in their location parameter, as a separate component of the parameter without any scaling coefficient, result in narrower return level confidence intervals and therefore tend to reduce uncertainty in extrapolated extremes.
\end{abstract}

Keywords: wavelet transform; statistically significant periodicities; non-stationary point process; uncertainty

\section{INTRODUCTION}

Within a general framework of assessing risk of coastal flooding, uncertainty analysis is nowadays regarded as a crucial component in the decision-making process. A possible reduction of uncertainty in predicting extreme coastal events can be significantly beneficial for the arrangement of coastal activities, the development of strategic management plans in coastal areas, as well as the design of future flood protection structures or the upgrading of existing ones. Recent advances in statistical modeling have provided civil engineers with an increasing power for making decisions under uncertainty. Due to the fact that information involved in engineering problems is in most cases imprecise, insufficient and subject to change, reliance must be placed on the ability of the engineer to synthesize existing information and to develop tools and methods to effectively use the existing uncertain information. One of the main objectives of the present research is to investigate uncertainty reduction in predictions of extreme marine events when prominent and statistically significant nonstationary features of the signals under study are included in the extreme analysis.

Morgan and Henrion (1990) present what is probably the best available taxonomy of types of uncertainty. In particular, they note that uncertainty can arise from a) Statistical variation e.g. random error in direct measurements of a quantity, b) Systematic error e.g. bias in the measuring apparatus and experimental procedure, c) Subjective judgment e.g. for quantities where empirical data is largely unavailable, d) Linguistic imprecision e.g. translation of verbal phrases into numerical probabilities, e) Variability e.g. quantities that vary over time or space, or from one person to another, f) Inherent randomness or unpredictability, which cannot be reduced by further research, g) Disagreement e.g. among multiple experts, h) Approximation e.g. due to limits in the spatial resolution of a model and i) Uncertainty about the most appropriate model to represent some phenomenon. According to van Gelder (1999) uncertainties in risk analysis can be divided in two categories: a) those that represent randomness in samples (inherent uncertainty) and b) those that come from basic knowledge of fundamental processes (epistemic uncertainty). Inherent uncertainties are subdivided in inherent uncertainties in space and in time and it is not possible for them to be reduced. Epistemic uncertainties are caused by lack of knowledge of all the causes and effects in physical systems or by lack of sufficient data and they may change as knowledge increases. Epistemic uncertainty includes model and statistical uncertainty. The later can be divided in parameter uncertainty and distribution type uncertainty.

The notion of uncertainty is closely related to extreme events associated with high return periods. Modern Extreme Value Theory (EVT), developed in the recent few decades, is regarded as the most promising approach to predict extreme events. EVT relies on the assumption that the limiting models suggested by the asymptotic theory continue to hold at finite but extreme levels. Nevertheless, a crucial

\footnotetext{
${ }^{1}$ Hydraulics Laboratory, Department of Civil Engineering, A.U.Th, University Campus, Thessaloniki, 54124, Greece
} 
assumption in fitting distribution functions to data is that the data are independent and identically distributed. The basic theoretical framework of extreme value models and inferential techniques can be found in Coles (2001). Models for block maxima and excesses over high thresholds (Peaks Over Threshold - POT approaches) are used, according to data availability, to extract design values for different variables. The "Peaks-Over-Threshold (POT)" model is described by the Generalized Pareto Distribution (GPD) with Poisson arrival rate and is considered advantageous compared to more traditional techniques of extremal analysis. Among others, Smith (1989), Coles and Tawn (1996), Pandey et al. (2001) and Egozcue et al. (2005) have contributed considerably to the use of the model in various applications. Recent studies on extreme value analysis for variables associated with the marine and coastal environment have been published by different researchers within the EU Research Project FLOODsite (www.floodsite.net). Sánchez-Arcilla et al. (2008) present their results based on a wide range of models, parameters and fitting techniques to a variety of geographic and climatic coastal, estuarine and riverine environments. They focus on selecting models and techniques based on a parsimony principle, to propose robust extrapolations of characteristic variables for a reliable flood hazard assessment. Van Gelder and Mai (2008) present an overview of different methods and techniques used to determine extreme values of river and sea related variables. Applications include the prediction of extreme water discharges in northwestern and central Europe and extreme waves along the Dutch North Sea coasts. Callaghan et al. (2008) perform a statistical simulation of extreme values of the wave climate in selected areas of the Australian coast using techniques of the univariate EVT.

It should be emphasized that most environmental variables exhibit phenomena of non-stationarity and more specifically that their observed variability can be characterized by a non-stationary stochastic process with a few periodic or nearly periodic components acting within time scales that range from the annual, over decadal, centennial, millennial or longer time periods. Therefore, incorporating covariate effects within EV models can simulate a part of the studied series variability and can also lead to unbiased estimates of return values of the variables. In the context of environmental processes non-stationarity is often apparent because of long-term trends and seasonality. Méndez et al. (2006) and Menéndez et al. (2008) study the long-term variability of extreme wave heights in the Pacific Ocean and present models to simulate it properly. Coles and Tawn (2005a) and Huerta and Sanso (2007) present EV models which include seasonal components by means of covariate modeling of the model parameters. Méndez et al. (2008) introduce a time-dependent POT model for extremes of significant wave height, conditioning to the duration of the storm and accounting for seasonality, while Menéndez et al. (2009) develop a time-dependent GEV model for monthly wave height maxima using harmonic functions in the model parameters. Galiatsatou and Prinos (2011) combine a non-stationary point process model and the continuous wavelet transform to model extreme wave heights in the Aegean Sea, trying to incorporate statistically significant seasonal features of the signals in the EV analysis.

The wavelet transform is a signal processing technique, very rapidly evolving in the recent years due to its ability to handle non-staionary signals and to provide immediate access to information that can be obscured by other time-frequency methods like the Fourier analysis. Numerous applications of the wavelet transform can be found in Torrence and Compo (1998), Massel (2001), Liu and Babanin (2004), Markovic and Koch (2005), Prinos et al. (2010) and many others. In the present study the wavelet transform is combined with non-stationary statistical models for extreme value analysis, to provide more reliable and more accurate return level estimates for storm surge and wave height extremes at the Dutch coast.

The methodology developed in the present paper is applied to three hourly measurements of storm surge and wave height in a selected location of the North Sea for a time period of 23 years. The wavelet transform has a dual role in the present work. First, it is used to detect the significant "periodicities" of the signals by means of the wavelet global and scale-averaged power spectra and then it is used to reconstruct the part of the time series, represented by these significant and prominent features. A non-stationary point process is utilized to model the extremes. A time varying threshold $u(t)$ with a period of one year and having an approximately uniform crossing rate throughout the year is used. The reconstructed part of the series variability representing the significant non-stationarities of each signal is incorporated in the location parameter of the point process model, together with selected harmonic functions, formulating a number of candidate extreme value models. The quality of the fitted models is assessed by means of the AIC (Akaike Information Criterion), as well as by means of diagnostic quantile plots. 


\section{METHODOLOGY}

Most environmental signals exhibit phenomena of non-stationarity. Non-stationarity is generally thought to indicate an underpinning driving force, regime or phase change(s) in the dynamics that generate the observed sequence/ signal. Trending behavior and seasonality are types of nonstationarity, which most commonly appear in time series. In maritime data non-stationarity is often present in the form of seasonal variations-effects. If a process is strongly seasonal, high values (or low values) appear constantly during specific periods of the year (the winter or the summer period). Wave heights, as well as storm surges, for example, tend to be higher in the winter than in the summer period. In the present work, a signal processing technique, namely the continuous wavelet transform is used at first to identify the main frequencies present in the data and therefore the dominant nonstationarities of the studied signals.

The continuous wavelet transform is a linear transform that decomposes an arbitrary signal $x(t)$ via basis functions with compact support that are simply dilations and translations of the parent wavelet (Kijewski-Correa and Kareem, 2007). The wavelet coefficients provide a measure of the similitude between the dilated/ shifted parent wavelet and the signal at time $t$ and scale $s$. The continuous wavelet transform of a discrete sequence $x_{n}$ is defined as the convolution of $x_{n}$ with a scaled and translated version of the mother (parent) wavelet (Torrence and Compo, 1998), forming the wavelet coefficients $W_{n}(s:$

$$
W_{n}(s)=\sum_{n^{\prime}=0}^{N-1} x_{n^{\prime}} \quad \psi^{*}\left[\frac{\left(n^{\prime}-n\right) \delta_{t}}{s}\right]
$$

where $\psi^{*}$ is the complex conjugate of the wavelet $\psi$, the variable $s$ is the scale of the wavelet transform, $\delta_{t}$ is the equal time spacing of the observations of $x_{n}, n=0,1, \ldots . N-1$ is the localized time index of the time series and $N$ is the number of points in the time series.

The wavelets are complex or real functions concentrated in time and frequency. Most wavelets are complex, which implies that the wavelet transform $W_{n}(s)$ is also complex. If a wavelet transform is complex, it can be divided into its real and its imaginary part. One of the most extensively used mother wavelets is the Morlet wavelet:

$$
\psi_{0}(\eta)=\pi^{-1 / 4} e^{i \omega_{0} \eta} e^{-\eta^{2} / 2}
$$

where $\eta$ is a non-dimensional time parameter and $\omega_{0}$ is the non-dimensional frequency. According to Farge (1992), for the Morlet wavelet to satisfy the admissibility condition, this frequency should be equal to 6. Eq. 2 represents a plane wave of frequency $\omega_{0}$ modulated by a Gaussian envelope (Massel, 2001). The Morlet wavelet is a common non-orthogonal complex wavelet. For the Morlet wavelet, the scale, $s$, and the Fourier period are nearly identical. Another wavelet commonly used is the Mexican hat wavelet, which is derived from a function proportional to the second derivative function of the Gaussian probability density function:

$$
\psi_{0}(\eta)=\left(\frac{2}{\sqrt{3}} \pi^{-1 / 4}\right)\left(1-\eta^{2}\right) \mathrm{e}^{-\eta^{2} / 2}
$$

The Mexican hat wavelet is a real valued wavelet function and does not have a scaling function, implying a non-orthogonal analysis. For the Mexican hat, the Fourier period is four times larger than the scale, $s$. The wavelet transform should reflect the features present in the signal. For time series with sharp steps, a boxcar-like wavelet should be selected, while for smoothly varying time series a smooth function is more appropriate (Massel, 2001).

The squared magnitude of the wavelet coefficients $\left|W_{n}(s)\right|^{2}$ can be presented as energy content in frequency and time and is called wavelet power spectrum. The wavelet power spectrum describes the time series variance at a selected scale (period) and at a selected moment in time (Torrence and Webster, 1999). To compare different wavelet power spectra, a normalization is performed, dividing the wavelet power spectrum by $\sigma^{2}$, giving a measure of the power relative to white noise. The scaleaveraged wavelet power spectrum is used to examine fluctuations in power over a range of scales. It is 
obtained by averaging the local wavelet coefficients along the $N$-vertical cuts of the time axis for a range of scales from $s_{1}$ to $s_{2}$ (Markovic and Koch, 2005):

$$
\bar{W}_{n}^{2}=\frac{\delta_{j} \delta_{t}}{C_{\delta}} \sum_{j=j_{1}}^{j=j_{1}} \frac{\left|W_{n}\left(s_{j}\right)\right|^{2}}{s_{j}}
$$

where $\delta_{j}$ depends on the width in spectral-space of the wavelet function, while $C_{\delta}$ is a constant for each wavelet function. The scale-averaged wavelet power can be viewed as a time series of the average variance in a certain band of scales. The average of the wavelet power over all local wavelet spectra along the time axis is the global wavelet power spectrum (Torrence and Compo, 1998):

$$
\bar{W}^{2}(s)=\left.\frac{1}{N} \sum_{n=0}^{N-1} W_{n}(s)\right|^{2}
$$

The wavelet power spectrum, the global wavelet spectrum and the scale-averaged wavelet spectrum can provide the main fluctuations in power of the studied signals at different scales and over a range of scales at different moments in time. This information can constitute the basis to perform reconstruction of the part of the signal indexed by these "periodicities".

The assembling of the wavelet components back into the original signal without loss of information is called reconstruction or synthesis. Reconstruction of the original time series is straightforward for the orthogonal wavelet transform, but it is complicated for the continuous wavelet transform by the redundancy in space and time. The delta function can be used to reconstruct the time series in case of continuous wavelet transform (Farge, 1992). By summing over a subset of scales, a wavelet filtered time series can be constructed (Torrence and Compo, 1998). For scales in the range $j_{l}$ to $j_{2}$, the wavelet filtered time series is:

$$
x_{n}^{\prime}=\frac{\delta_{j} \delta_{t}^{1 / 2}}{C_{\delta} \psi_{0}(0)} \sum_{j=j_{1}}^{j=j_{z}} \frac{\left\{W_{n}\left(s_{j}\right)\right\}}{s_{j}^{1 / 2}}
$$

For the Morlet wavelet $\psi_{0}(0)=\pi^{-(1 / 4)}$ and for the Mexican hat $\psi_{0}(0)=0.867$. The constant $C_{\delta}$ is $C_{\delta}=$ 0.776 for the Morlet wavelet and $C_{\delta}=3.541$ for the Mexican hat.

In the present work a non-stationary point process model, namely the Poisson process is used to model extremes of wave heights and storm surges incorporating the effects of non-stationarity in the extremal analysis. Within the point process framework, seasonality and non-stationarity in general, can be expressed using an intensity function, proposed by Smith (1989):

$$
\lambda_{\boldsymbol{\theta}}(x)=\frac{1}{\sigma(t)}\left\{1+\xi(t)\left(\frac{x-\mu(t)}{\sigma(t)}\right)\right\}_{+}-(\xi(t)+1) / \xi(t)
$$

where $0<t<n_{y}, \quad x>u(t)$ and $\mu(t), \sigma(t), \xi(t)$ are parametric functions of time and $u(t)$ is a time dependent threshold. Time dependent thresholds are very important in case of strongly seasonal processes. When such phenomenon is observed, constant thresholds will generate more exceedances in the winter than in the summer, leading to an unbalanced bias-variance trade off over the year. In this work seasonality of the wave and storm surge data is modelled using a time varying threshold $u(t)$ with a period of one year, following the methodology of Coles and Tawn (2005b). The year is divided in a winter (October-March) and a summer (April-September) period and the threshold is specified as:

$$
u(t ; a, b)=a+b \cos (2 \pi t)
$$

where $t$ measures the time (years) and Eq. 8 corresponds to an annual cycle peaking at the $1^{\text {st }}$ January and having its minimum values during summer. Parameters $a$ and $b$ are chosen so as to obtain a fixed uniform crossing rate over the winter and summer periods by minimization of the function: 


$$
h(a, b)=\max \left\{\left(p_{s}(a, b)-q\right)^{2},\left(p_{w}(a, b)-q\right)^{2}\right\}
$$

where $p_{s}(a, b)$ and $p_{w}(a, b)$ are the proportions of exceedances of the threshold $u(t ; a, b)$ in the summer and winter periods, respectively and $q$ is the desired uniform crossing rate. The parameters $a$ and $b$ can be interpreted respectively as the median threshold level and the scale of variation between peak summer and winter periods.

Regarding the parameters of the non-stationary point process, two different categories of models will be utilized. The first one includes simple sinusoidal harmonics in the location and/ or in the scale parameters of the EV model. The shape parameter is kept constant. The location and the scale parameters of this category can be represented as:

$$
\begin{gathered}
\mu(t)=\mu_{0}+\sum_{i=1}^{p_{\mu}} \mu_{2 i-1} \cos (i 2 \pi t)+\mu_{2 i} \sin (i 2 \pi t) \\
\sigma(t)=\exp \left(\sigma_{0}+\sum_{i=1}^{p_{\sigma}} \sigma_{2 i-1} \cos (i 2 \pi t)+\sigma_{2 i} \sin (i 2 \pi t)\right)
\end{gathered}
$$

where the time $t$ is measured in years, $\mu_{0}$ and $\sigma_{0}$ are the mean values, $\mu_{i}$ and $\sigma_{i}$ are the amplitudes of the harmonics and $p_{\mu}$ and $p_{\sigma}$ determine the number of harmonics per year. It should be noted that candidate models are also tested that do not include the sine function in their location and/ or scale parameter, due to the form of the selected threshold that does not contain a phase shift. The parameter vector $\boldsymbol{\theta}=$ $\left(\mu_{0}, \sigma_{0}, \mu_{i}, \sigma_{i}, \xi_{0}\right)$ of the model is estimated using MLE. In the present work, the largest parameterization used for this group of models has $p_{\mu}=2$ and $p_{\sigma}=2$. The second group of non-stationary point processes contains the reconstructed part of statistically significant signal features in the location and/or scale parameters. The shape parameter is again kept constant. The location and the scale parameters of this category of models can be represented as:

$$
\begin{gathered}
\mu(t)=\mu_{0}+a \mu_{w}(t)+\sum_{i=1}^{p_{\mu}^{\prime}} \mu_{2 i-1} \cos (i 2 \pi t)+\mu_{2 i} \sin (i 2 \pi t) \\
\sigma(t)=\exp \left(\sigma_{0}+\beta \mu_{w}(t)+\sum_{i=1}^{p_{\sigma}^{\prime}} \sigma_{2 i-1} \cos (i 2 \pi t)+\sigma_{2 i} \sin (i 2 \pi t)\right)
\end{gathered}
$$

where $\mu_{w}(t)$ is the component of the signal produced using the inverse wavelet transform (reconstruction process), $p_{\mu}^{\prime}$ and $p_{\sigma}^{\prime}$ determine the number of harmonics per year, and $\alpha$ and $\beta$ are coefficients that are either fixed to $\alpha=1$ and/or $\beta=0$, or estimated together with the other parameters using MLE. As for the first category of statistical models, some of the candidate models tested do not include the sine function in their location and/ or scale parameter. The largest parameterization of the harmonic functions used in the present work is $p_{\mu}^{\prime}=1$ and $p_{\sigma}^{\prime}=1$ to represent the annual cycle and to comply with the annually periodic threshold used.

For the non-stationary version of the point process model, return levels can still be estimated, though the precise form depends on the model for non-stationarity. If $z_{m}$ is the $m$-years return level and $n_{y}$ is the number of observations per year, for the special case of considering seasonality over a oneyear cycle:

$$
\sum_{i=1}^{\mathrm{n}_{v}} \log p_{\mathrm{i}}=\log (1-1 / m), p_{i}=1-n^{-1}\left[1+\xi_{i}\left(z_{m}-\mu_{i}\right) / \sigma_{i}\right]^{-1 / \xi_{i}} \text { if }\left[1+\xi_{i}\left(z_{m}-\mu_{i}\right) / \sigma_{i}\right]>0 \text { otherwise } p_{i}=1
$$

which can be easily solved for $z_{m}$ using standard numerical methods for non-linear equations (Coles, 2001). Confidence intervals for "aggregated" return levels can be computed by means of simulation. 
To perform the simulation, the distribution of the parameters of each candidate model is assumed to be multivariate Normal with mean values of the parameters equal to their maximum likelihood estimators and variance equal to the variance-covariance matrix of the ML estimation. Simulating the model parameters vector from this distribution, $k$ values of $\boldsymbol{\theta}$ are produced, namely $\boldsymbol{\theta}_{1}, \boldsymbol{\theta}_{2}, \ldots, \boldsymbol{\theta}_{k}$, and these values are then substituted in Eq. 12 to obtain approximate $95 \%$ confidence intervals.

However, in a non-stationary context the usual interpretation of return levels with probability of exceedance $1 / p$ is no longer valid and it is better to think of it as a quantile of the distribution of the variable under study in the current year. Therefore "local" return levels can be used in such cases. For a constant shape parameter $\xi \neq 0$, the time-dependent extreme quantiles associated with return periods of $R$ years are estimated as:

$$
x_{R}(t)=\mu(t)-\frac{\sigma(t)}{\xi}\left\{1-\left[-\log (1-1 / R)^{-1 / \xi}\right\}\right.
$$

Confidence intervals for "local" return level estimates can be computed using the delta method, assuming normality of the maximum likelihood estimators.

The quality of the fit of different candidate models can be judged using the Akaike Information Criterion (AIC). The AIC is a penalty function that is used to compare models that come from the same parent distribution:

$$
A \mathrm{IC}=-2 l(\boldsymbol{\theta})+2 p
$$

where $l(\boldsymbol{\theta})$ is the maximum log-likelihood resulting from each candidate model and $p$ is the number of parameters of the model estimated by MLE. The model that minimizes the AIC is the simplest one that provides a good fit to the data. The goodness of fit of the different models is also assessed my means of quantile plots. When time-dependent variables are used, to construct appropriate diagnostic plots the data are usually transformed to the frequency, $Z$, and intensity, $W$, statistics introduced by Smith (1989). The two statistics are estimated at time $t_{k} \in\left[t_{1}, t_{n}\right]$ as:

$$
\begin{gathered}
Z_{k}={ }_{{ }^{t} k-1}^{{ }^{t}} \hat{\ell}_{u}(t) d t, \lambda_{u}(t)=\left[1+\xi(t) \frac{(u(t)-\mu(t))}{\sigma(t)}\right]^{-1 / \xi(t)} \\
W_{k}=\frac{1}{\xi\left(t_{k}\right)} \log \left[1+\frac{\xi\left(t_{k}\right) y_{k}}{\sigma\left(t_{k}\right)+\xi\left(t_{k}\right)\left(u\left(t_{k}\right)-\mu\left(t_{k}\right)\right)}\right]
\end{gathered}
$$

If the model is correct the $Z$, as well as the $W$ variables for all $k$ should be independent exponential random variables with mean 1. Quantile plots can be produced for both $Z$ and $W$. Since the hypothesized distribution $G$ is unit exponential, for which $G^{-1}(p)=-\log (1-p)$, this means plotting either $Z_{i: n}$ or $W_{i: n}$ (the $i^{\text {th }}$ smallest of $n$ ordered values) against $-\log \left(1-p_{i: n}\right)$ where $p_{i: n}=(i-0.5) / n$.

\section{ANALYSIS OF RESULTS}

In the present work wave height and storm surge data from the Dutch coast are used to test the efficiency of the proposed methodology. More specifically, the Dutch station Swb (Schouwenbank), located in a depth of $15 \mathrm{~m}$ in the Dutch part of the North Sea is selected. The data files available for Swb contain three hourly data of wave height $H_{m 0}$ and its standard deviation, average wave period $T_{m 02}$, main wave direction $T_{h 0}$, average wave height and period of the highest third part $H_{1 / 3}$ and $T_{H 1 / 3}$ and the wave height of the low frequency waves $H_{T E 3}$. In addition to the wave parameters, the files also include data on wind speed and direction, water level relative to NAP/MSL, set-up or storm surge and a column indicating the origin of the measured variables. These values are always calculated from samples lasting 20 minutes, but they are supposed to represent a 3-hour period. The datasets consist of a sequence of 23 years over the period 1979-2001. Two wave survey instruments, namely a main sensor and a secondary sensor, were used to perform the measurements. If both sensors have registered values for a parameter, then the mean value is given. If, however, data are only present from one sensor, then this is used in the file and if neither of the sensors have values, then estimated values are 
retained. Missing records are patched by hindcasting. It should be noted that at most wave sites in the North Sea, waves are only measured, not wind or water level. The data for wind and water level originate from other neighbouring locations. In the present paper, from all available datasets, three hourly measurements of significant wave height $\left(H_{1 / 3}\right)$ and storm surge are utilized.

The first stage of the proposed methodology includes the implementation of the wavelet transform to the available datasets of wave height and storm surge. To construct the wavelet transform of both series, the mean value of the entire record is removed, since the wavelet transform is mainly used to isolate the timescales of strong variability of the process under study. To reduce the wraparound effects, the time series is padded with zeros. To perform the wavelet analysis of both time series two non-orthogonal wavelet functions, namely the Morlet and the Mexican hat are used in the present work. The most noticeable difference between the two wavelet functions is the fine scale structure resulting utilizing the latter one. It should be noted that regarding the wavelet power spectrum, the same features appear when both wavelet functions are implemented, approximately at the same locations and with similar power. Therefore for the sake of brevity in the present work results are presented for storm surges by means of the Morlet and for wave heights using the Mexican hat wavelet functions. For both time series, results using the two wavelet functions do not differ significantly. Figures 1 and 2 present the normalized wavelet power spectrum for storm surges and wave heights at station Swb, respectively. As mentioned earlier, for storm surge data, the complex Morlet wavelet is utilized to construct the spectrum of Fig. 1, while for wave height data the wavelet power spectrum (Fig. 2) is constructed using the real-valued Mexican hat wavelet. It should be noted that the power spectrum is given as a base-2 logarithm of wavelet power. The broken line in both Figures represents the Cone of Influence (COI) of the wavelet transform, a region of the spectrum in which edge effects become important and oscillations can possibly be artifacts of padding the time series with zeros.

From Figures 1 and 2 it is evident that there exists a high wavelet power concentration near the 1year band for both storm surges and wave heights along most of the time series length. Comparing the two Figures, the main difference between the two wavelet functions can be conceived. For the Morlet wavelet the wavelet power spectrum combines both positive and negative peaks into a single broad peak, while the Mexican hat captures positive and negative oscillations as separate peaks (Torrence and Compo, 1998). Therefore, the non-stationarity present near the 1-year band, which can be attributed to seasonal effects due to different climate patterns in different months, is presented with a broad wavelet power concentration for the Morlet and with separate highs and lows for the Mexican hat wavelet. For the latter, seasonality is better illustrated, because the highest values of the wavelet coefficients are observed at the beginning and at the end of a year during the winter period, while the lowest ones are observed during summer. Taking into account that the storm surge and wave height time series have the same length, it should also be noted that the Mexican hat has a narrower COI.

Another prominent feature of the wavelet power spectrum for storm surges is the existence of high variability in the [2,8]-years band of scales. These oscillations can be possibly attributed to the influence of the North Atlantic Oscillation (NAO), which is a mode of large-scale variability within the North Atlantic. Butler (2005) has shown that the NAO indices exhibit variability at a number of different temporal scales, including year-to-year variations, short-term and decadal variations and has also studied and proven the existence of a relationship between the NAO and extreme storm surge characteristics.

Figure 3(a, b) presents the global wavelet power spectrum for both the storm surge and the wave height time series at station Swb. To test the statistical significance of the spectrum peaks, a theoretical red noise spectrum is defined. The AR(1) model is used to simulate this spectrum, with autocorrelation factor estimated directly from the datasets. After defining the univariate lag-1 autoregressive model, the background spectrum for red noise is multiplied by the $95^{\text {th }}$ percentile value for $\chi_{2}^{2}$, to determine the $5 \%$ significance level $(95 \%$ confidence level) of the wavelet global spectrum (Torrence and Compo, 1998). The autocorrelation coefficients for storm surge and wave height series are estimated as $a_{\mathrm{ss}}=0.84$ and $a_{\mathrm{w}}=0.95$, respectively. 


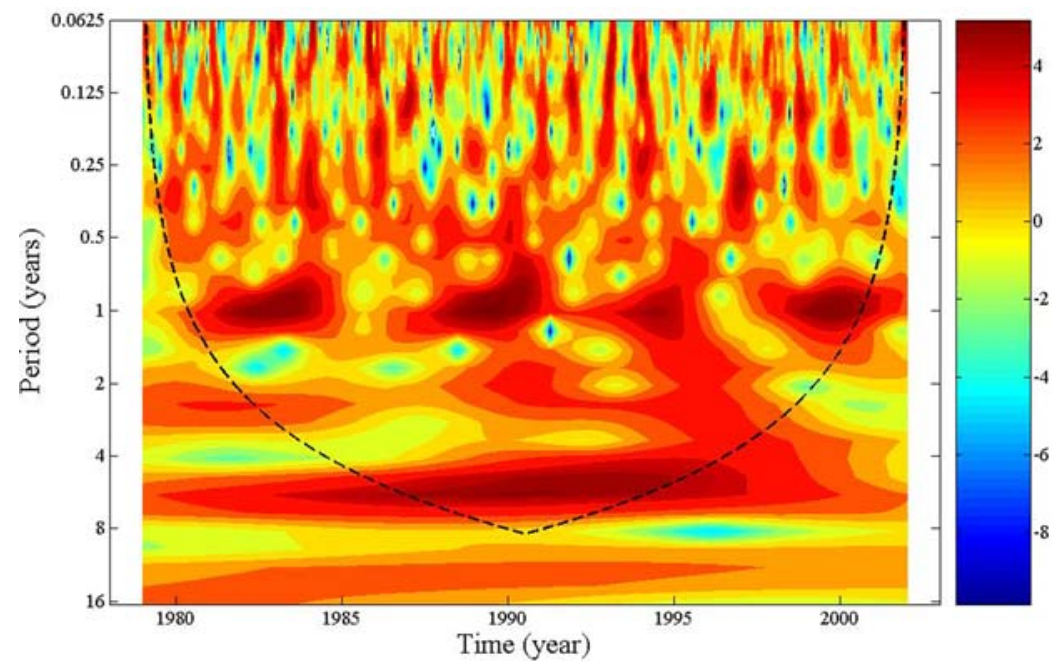

Figure 1. Normalized wavelet power spectrum for storm surges at station Swb using the Morlet wavelet and the COI (dashed line)

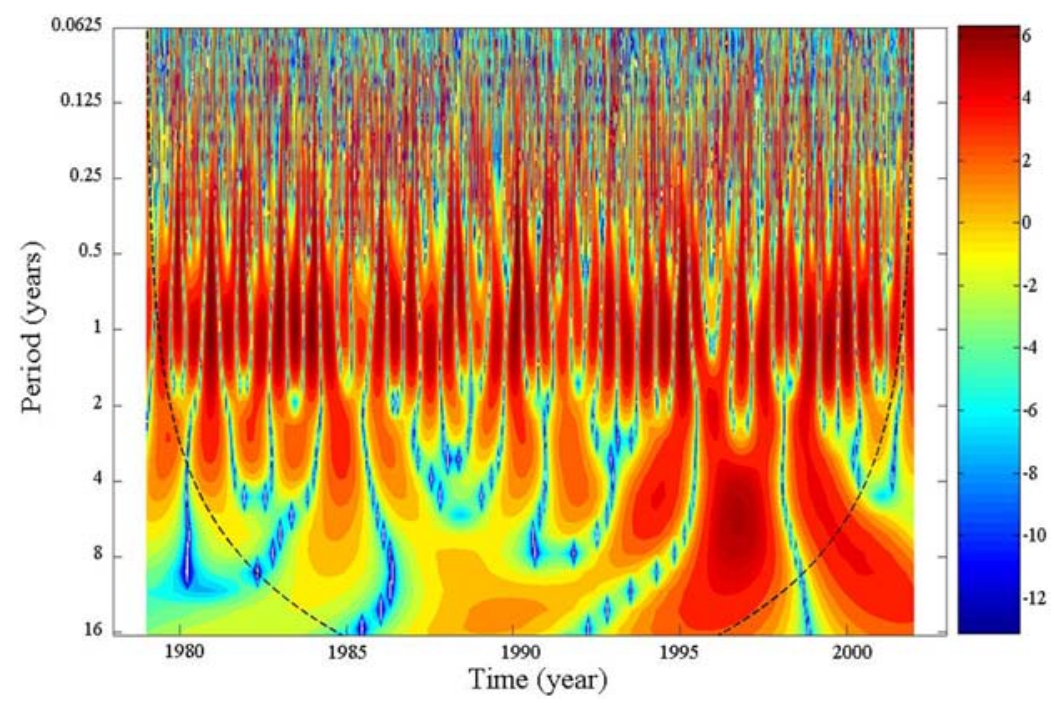

Figure 2. Normalized wavelet power spectrum for wave heights at station Swb using the Mexican hat wavelet and the COI (dashed line)
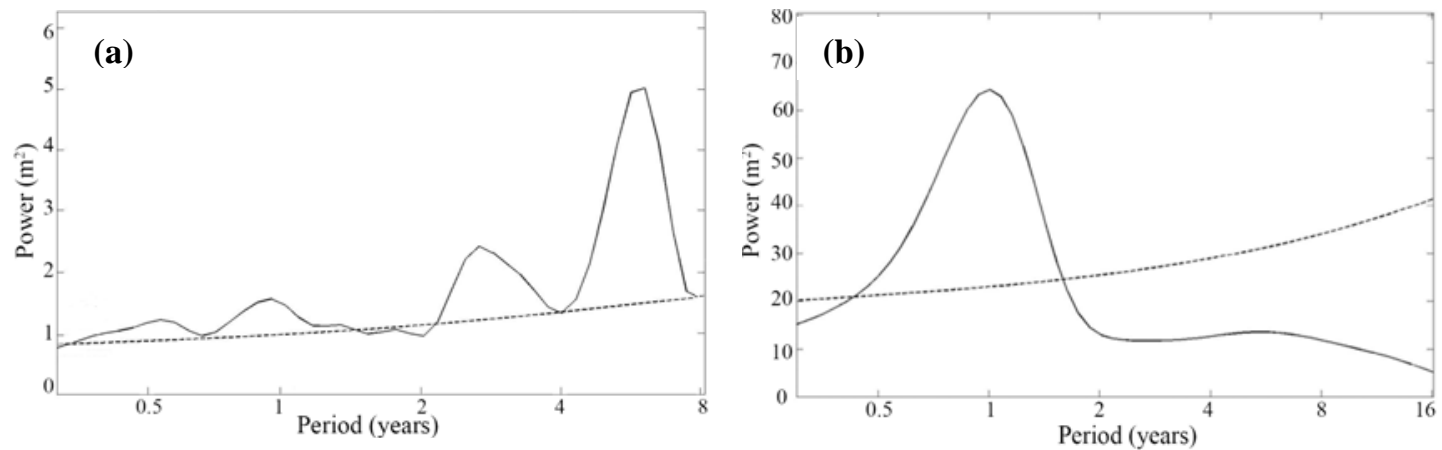

Figure 3. The global wavelet spectrum (continuous line) for: a) storm surges using the Morlet wavelet, b) wave heights using the Mexican hat wavelet and the associated $95 \%$ confidence levels (dashed line) 
From Figure 3(a) it is evident that the global wavelet spectrum for the storm surge data presents statistically significant peaks at a 5\% significance level near the periods of 0.5 and 1 years, as well as in the intervals of 2-4 and 4-8 years. However, although the most prominent peak of the global wavelet spectrum lies within the $[4,8]$ years band, these oscillations are ignored in the rest of the analysis, because of the fact that most of the time series variability within this interval is included in the COI (Fig. 1). Therefore periods in the range of $[0.3,1.6]$ and $[2.2,4]$ years are considered statistically significant for storm surges at Swb. For wave heights (Fig. 3(b)), there is one single evident peak near the 1 -year band. The statistically significant periodicities at a $5 \%$ significance level lay within the interval $[0.35,1.7]$ years. Comparing the two spectra in terms of the wavelet functions used, the peaks for the Mexican hat wavelet appear more outspread, because the Mexican hat is broader in spectral space than the Morlet.

A measure of the average variance of a series versus time within a range of scales is given by the scale-averaged wavelet power. Figure $4(a, b, c)$ presents the scale-averaged wavelet power spectrum over the identified statistically significant scales at a 5\% significance level (continuous line) for storm surges (Fig. 4(a, b)) and wave heights (Fig. 4(c)), together with the respective 95\% confidence levels (dashed line). The variability of the storm surge signal in the range of scales $[0.3,1.6]$ and $[2.2,4]$ years (Fig. 4(a) and Fig. 4(b), respectively) seems to be statistically significant along almost half of the entire time period considered. For wave heights, the variability within the range of scales $[0.35,1.7]$ years (Fig. 4(c)) seems to present statistically significant peaks along the entire time series. The form of the scale-averaged wavelet spectrum of the Mexican hat was expected by the shape of the local wavelet spectrum (Fig. 2), characterized by a narrow structure in time-space and low values of wavelet power during some periods of the year.
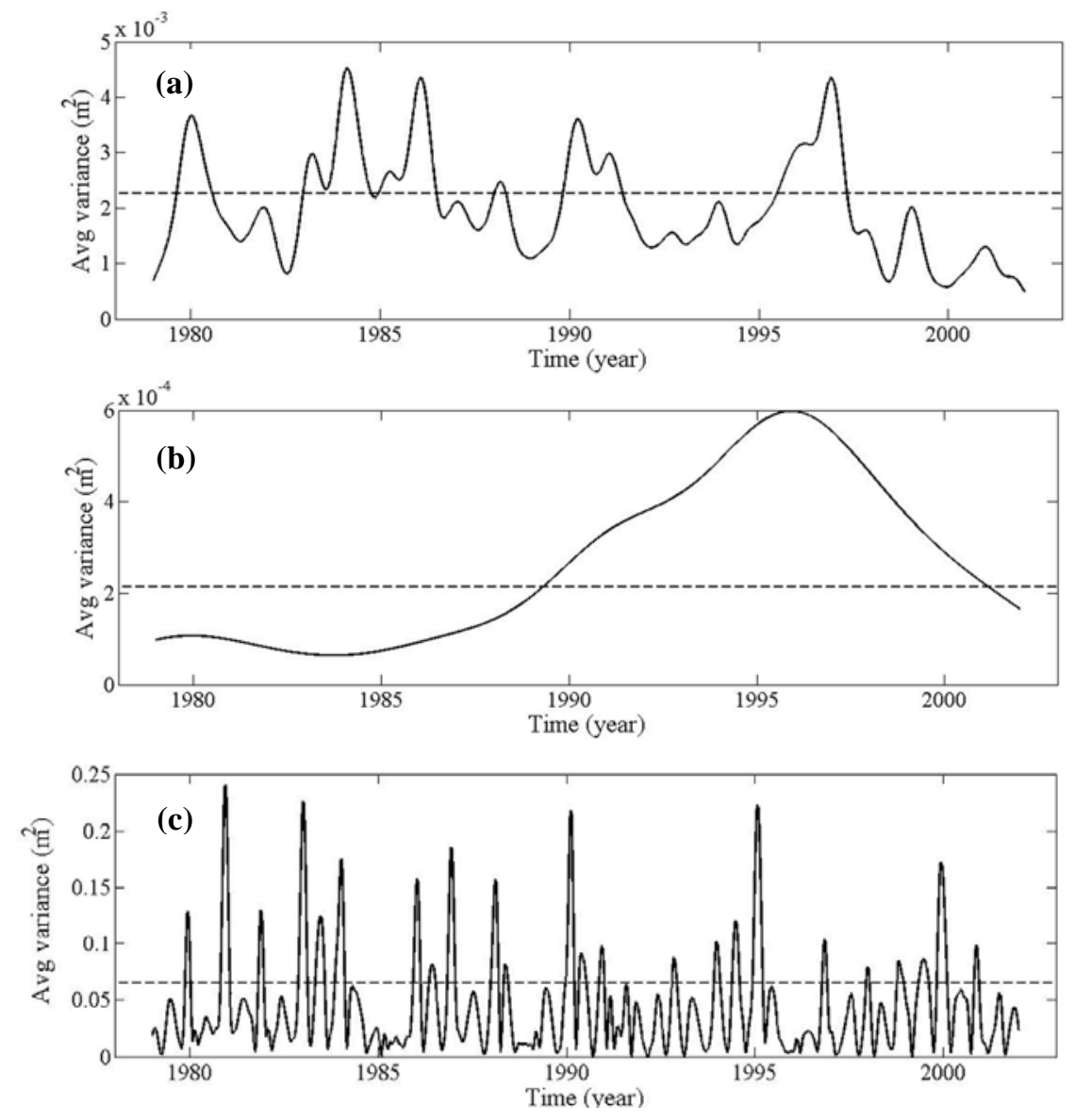

Figure 4. The scale-averaged wavelet spectrum (continuous line) for: a) storm surge periods in the range [0.3, 1.6] years using the Morlet wavelet, b) storm surge periods in the range [2.2, 4] years using the Morlet wavelet c) wave height periods in the range $[0.35,1.7]$ years using the Mexican hat wavelet and the associated $95 \%$ confidence levels (dashed line) 
The global wavelet spectrum and the scale-averaged wavelet spectrum can provide the main fluctuations in power of the signal at different scales and over a range of scales at different moments in time. This information can constitute the basis to perform reconstruction of the part of the signal indexed by these "periodicities". Figures 5 and 6 present the reconstructed part of the storm surge and wave height without the mean value, for the range of periods $[0.3,1.6]$ years and $[0.35,1.7]$ years, respectively, using the Morlet and the Mexican hat wavelets. Points (crosses) in both Figures represent the observations of storm surge and wave height at station Swb, without the mean value of the series. The reconstructed part of storm surge data in the range of periods $[2.2,4]$ years, although included in the analysis that follows, is not presented here for the sake of brevity.

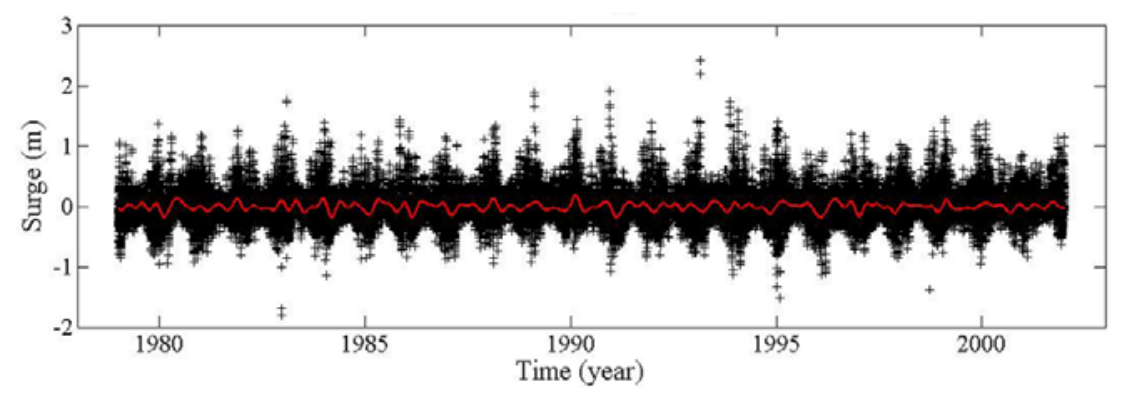

Figure 5. Reconstructed part of the storm surge series at station Swb for periods in the range $[0.3,1.6]$ years using the Morlet wavelet (red line) and original data (crosses) without the mean value.

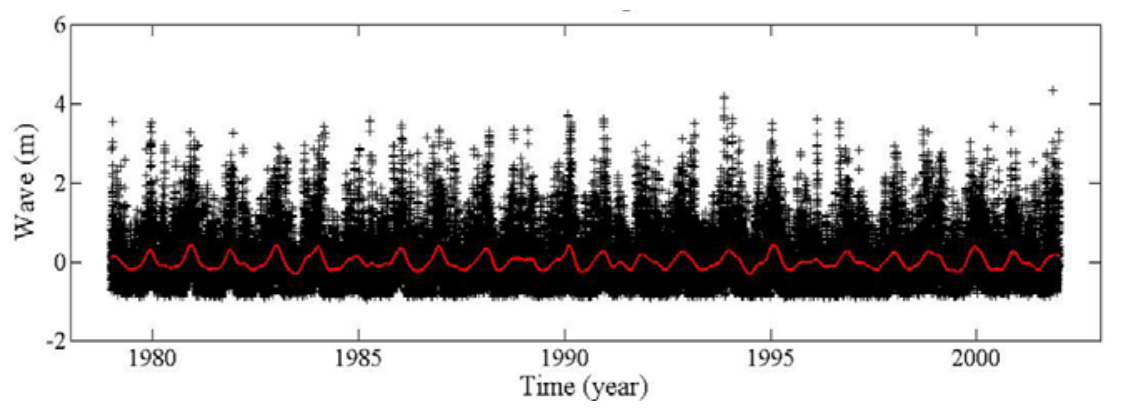

Figure 6. Reconstructed part of the wave height series at station Swb for periods in the range $[0.35,1.7]$ years using the Mexican hat wavelet (red line) and original data (crosses) without the mean value.

The application of EVT assumes independence of successive extreme observations. However, extreme events are typically found during storms, lasting for hours or even days (Zachary et al. 1998). It is therefore important to consider the effect of such short-term dependence in the data. Previous studies of offshore data use period lengths between 24 and 60 hours to select independent observations in a rather simple way. Galiatsatou (2009) has proven that a time interval of $24 \mathrm{~h}$ is adequate for both storm surge and wave height data at the Dutch part of the North Sea. Therefore daily storm surge and wave height maxima are selected to apply the proposed methodology. Most of the successive maxima in both storm surge and wave height series are not supposed to be caused by the same storm events. But, there also exist daily maxima of the original three hourly observations that cluster at the boundaries between successive periods, but the effect of this is relatively minor and has a small probability within the series considered and it should be emphasized that its effects with respect to return levels and return periods is conservative.

The non-stationary point process described by Eq. 7 with time varying location and scale parameters and a time varying threshold is utilized in the present work. The time varying threshold $u(t)$ has a period of one year and is characterized by a uniform crossing rate within the year. The constant rate used for both variables is set to $q=0.05$. This threshold is define as $u=0.62+0.28 \cos (2 \pi t)$ and $u=2.86+0.66 \cos (2 \pi t)$ for storm surge and wave height daily maxima, respectively, where $t$ is measured in years.

Having specified the time varying threshold for both variables, the two categories of non-stationary point process models presented in the previous section are fitted to the daily maxima using the procedure of MLE. The performance of all different models belonging to the same category is judged 
by means of the AIC defined in the previous section. After estimating the model parameters, return level estimates are extracted for both storm surges and wave heights corresponding to return periods of 100,1000 and 10000 years for all different distribution functions assessed. The return period of 10000 years is very large for the return level estimates to be reliable, considering the fact that the sample size for both marine variables is limited to 23 years. However, results are also presented for this return period, because it is used to estimate flooding risk, as well as for designing purposes in parts of the Dutch coast.

Tables 1 and 2 present "aggregated" return level estimates for storm surge and wave height data, respectively, corresponding to return periods of 100, 1000 and 10000 years using Eq. 12 for representative models of the first and second category analyzed earlier. In Tables 1 and 2, the median return level is presented together with its $95 \%$ confidence interval (lower $2.5 \%$ and upper $97.5 \%$ confidence intervals). Despite the fact that Eq. 12 is used to estimate return levels for the special case of considering seasonality over a 1-year cycle, it is used here to provide an approximate estimate of return level confidence intervals for a representative year of the samples. The concentration of the wavelet power near the 1-year band, resulting in a peak period of one year partly justifies the approximate use of this equation.

Table 1. "Aggregated" return level estimates (median and 95\% confidence intervals) of storm surge for return periods of 100,1000 and 10000 years for representative models with harmonic functions or $\mu_{\mathrm{w}}(t)$ and harmonic functions in the parameters of the EV model, with associated AIC values.

\begin{tabular}{|c|c|c|c|c|}
\hline \multirow{2}{*}{ Model } & \multicolumn{3}{|c|}{ Storm Surge $(\mathrm{m})$} & \multirow{2}{*}{ AIC } \\
\hline & 100 years & 1000 years & 10000 years & \\
\hline (1a) $\mu=\mu_{0}, \sigma=\sigma_{0}$ & $\begin{array}{c}2.26 \\
(1.97,2.61) \\
\end{array}$ & $\begin{array}{c}2.58 \\
(2.16,3.12)\end{array}$ & $\begin{array}{c}2.84 \\
(2.29,3.62) \\
\end{array}$ & -1947.68 \\
\hline (1b) $\mu=\mu_{0}+\mu_{1} \cos (2 \pi t), \sigma=\exp \left(\sigma_{0}+\sigma_{1} \cos (2 \pi t)\right)$ & $\begin{array}{c}2.23 \\
(2.00,2.45) \\
\end{array}$ & $\begin{array}{c}2.59 \\
(2.28,2.96) \\
\end{array}$ & $\begin{array}{c}2.90 \\
(2.46,3.46) \\
\end{array}$ & -2250.69 \\
\hline $\begin{array}{l}(1 \mathrm{c}) \mu=\mu_{0}+\mu_{1} \cos (2 \pi t)+\mu_{2} \sin (2 \pi t), \\
\sigma=\exp \left(\sigma_{0}+\sigma_{1} \cos (2 \pi t)+\sigma_{2} \sin (2 \pi t)\right)\end{array}$ & $\begin{array}{c}2.18 \\
(1.98,2.44)\end{array}$ & $\begin{array}{c}2.51 \\
(2.25,2.94)\end{array}$ & $\begin{array}{c}2.79 \\
(2.41,3.41)\end{array}$ & -2251.12 \\
\hline $\begin{array}{l}\text { (1d) } \mu=\mu_{0}+\mu_{1} \cos (2 \pi t)+\mu_{2} \sin (2 \pi t)+\mu_{3} \cos (4 \pi t)+\mu_{4} \sin (4 \pi t) \\
\sigma=\exp \left(\sigma_{0}+\sigma_{1} \cos (2 \pi t)+\sigma_{2} \sin (2 \pi t) \sigma_{3} \cos (4 \pi t)+\sigma_{4} \sin (4 \pi t)\right)\end{array}$ & $\begin{array}{c}2.24 \\
(1.99,2.56) \\
\end{array}$ & $\begin{array}{c}2.58 \\
(2.22,3.14) \\
\end{array}$ & $\begin{array}{c}2.87 \\
(2.39,3.72) \\
\end{array}$ & -2247.56 \\
\hline (2a) $\mu=\mu_{0}+\mathrm{a} \mu_{\mathrm{w}}(t)+\mu_{1} \cos (2 \pi t), \sigma=\exp \left(\sigma_{0}+\sigma_{1} \cos (2 \pi t)\right)$ & $\begin{array}{c}2.23 \\
(2.02,2.48) \\
\end{array}$ & $\begin{array}{c}2.59 \\
(2.26,3.00)\end{array}$ & $\begin{array}{c}2.89 \\
(2.44,3.55)\end{array}$ & -2248.69 \\
\hline$(2 \mathrm{~b}) \mu=\mu_{0}+a \mu_{\mathrm{w}}(t)+\mu_{1} \cos (2 \pi t), \sigma=\exp \left(\sigma_{0}+\beta \mu_{\mathrm{w}}(t)+\sigma_{1} \cos (2 \pi t)\right)$ & $\begin{array}{c}2.22 \\
(2.02,2.44) \\
\end{array}$ & $\begin{array}{c}2.59 \\
(2.29,2.98) \\
\end{array}$ & $\begin{array}{c}2.91 \\
(2.49,3.52)\end{array}$ & -2247.08 \\
\hline (2c) $\mu=\mu_{0}+\mu_{\mathrm{w}}(t)+\mu_{1} \cos (2 \pi t), \sigma=\sigma_{0}$ & $\begin{array}{c}2.04 \\
(1.83,2.34) \\
\end{array}$ & $\begin{array}{c}2.41 \\
(2.06,2.95) \\
\end{array}$ & $\begin{array}{c}2.77 \\
(2.26,3.63) \\
\end{array}$ & -2180.64 \\
\hline$(2 \mathrm{~d}) \mu=\mu_{0}+\mu_{\mathrm{w}}(t)+\mu_{1} \cos (2 \pi t), \sigma=\exp \left(\sigma_{0}+\sigma_{1} \cos (2 \pi t)\right)$ & $\begin{array}{c}2.28 \\
(2.10,2.45) \\
\end{array}$ & $\begin{array}{c}2.62 \\
(2.36,2.92) \\
\end{array}$ & $\begin{array}{c}2.89 \\
(2.56,3.32) \\
\end{array}$ & -2233.41 \\
\hline$(2 \mathrm{e}) \mu=\mu_{0}+\mu_{\mathrm{w}}(t)+\mu_{1} \cos (2 \pi t), \sigma=\exp \left(\sigma_{0}+\beta \mu_{\mathrm{w}}(t)\right)$ & $\begin{array}{c}2.17 \\
(1.88,2.63) \\
\end{array}$ & $\begin{array}{c}2.71 \\
(2.19,3.66) \\
\end{array}$ & $\begin{array}{c}3.29 \\
(2.47,5.00) \\
\end{array}$ & -2202.23 \\
\hline
\end{tabular}

\begin{tabular}{|c|c|c|c|c|}
\hline \multirow[b]{2}{*}{ Model } & \multicolumn{3}{|c|}{ Wave Height (m) } & \multirow[b]{2}{*}{ AIC } \\
\hline & 100 vears & 1000 years & 10000 vears & \\
\hline (1a) $\mu=\mu_{0}, \sigma=\sigma_{0}$ & $\begin{array}{c}5.33 \\
(5.12,5.35) \\
\end{array}$ & $\begin{array}{c}5.49 \\
(5.24,5.76) \\
\end{array}$ & $\begin{array}{c}5.57 \\
(5.29,5.78)\end{array}$ & -1121.38 \\
\hline (1b) $\mu=\mu_{0}+\mu_{1} \cos (2 \pi t), \sigma=\exp \left(\sigma_{0}+\sigma_{1} \cos (2 \pi t)\right)$ & $\begin{array}{c}5.92 \\
(5.44,6.57) \\
\end{array}$ & $\begin{array}{c}6.42 \\
(5.72,7.42) \\
\end{array}$ & $\begin{array}{c}6.79 \\
(5.92,8.37) \\
\end{array}$ & -1416.20 \\
\hline $\begin{array}{l}(1 \mathrm{c}) \mu=\mu_{0}+\mu_{1} \cos (2 \pi t)+\mu_{2} \sin (2 \pi t) \\
\sigma=\exp \left(\sigma_{0}+\sigma_{1} \cos (2 \pi t)+\sigma_{2} \sin (2 \pi t)\right)\end{array}$ & $\begin{array}{c}5.98 \\
(5.49,6.67) \\
\end{array}$ & $\begin{array}{c}6.51 \\
(5.83,7.63) \\
\end{array}$ & $\begin{array}{c}6.93 \\
(6.00,8.55) \\
\end{array}$ & -1414.40 \\
\hline $\begin{array}{l}(1 \mathrm{~d}) \mu=\mu_{0}+\mu_{1} \cos (2 \pi t)+\mu_{2} \sin (2 \pi t)+\mu_{3} \cos (4 \pi t)+\mu_{4} \sin (4 \pi t), \\
\sigma=\exp \left(\sigma_{0}+\sigma_{1} \cos (2 \pi t)+\sigma_{2} \sin (2 \pi t) \sigma_{3} \cos (4 \pi t)+\sigma_{4} \sin (4 \pi t)\right)\end{array}$ & $\begin{array}{c}5.84 \\
(5.35,6.52) \\
\end{array}$ & $\begin{array}{c}6.32 \\
(5.65,7.39) \\
\end{array}$ & $\begin{array}{c}6.71 \\
(5.85,8.20)\end{array}$ & -1410.34 \\
\hline (2a) $\mu=\mu_{0}+a \mu_{\mathrm{w}}(t)+\mu_{1} \cos (2 \pi t), \sigma=\exp \left(\sigma_{0}+\sigma_{1} \cos (2 \pi t)\right)$ & $\begin{array}{c}5.92 \\
(5.41,6.57) \\
\end{array}$ & $\begin{array}{c}6.44 \\
(5.69,7.43) \\
\end{array}$ & $\begin{array}{c}6.82 \\
(5.88,8.23)\end{array}$ & -1414.42 \\
\hline$(2 \mathrm{~b}) \mu=\mu_{0}+a \mu_{\mathrm{w}}(t)+\mu_{1} \cos (2 \pi t), \sigma=\exp \left(\sigma_{0}+\beta \mu_{\mathrm{w}}(t)+\sigma_{1} \cos (2 \pi t)\right)$ & $\begin{array}{c}5.90 \\
(5.43,6.50) \\
\end{array}$ & $\begin{array}{c}6.46 \\
(5.78,7.41)\end{array}$ & $\begin{array}{c}6.91 \\
(6.05,8.30)\end{array}$ & -1413.24 \\
\hline (2c) $\mu=\mu_{0}+\mu_{\mathrm{w}}(t)+\mu_{1} \cos (2 \pi t), \sigma=\sigma_{0}$ & $\begin{array}{c}5.65 \\
(5.42,6.00)\end{array}$ & $\begin{array}{c}5.95 \\
(5.63,6.50)\end{array}$ & $\begin{array}{c}6.29 \\
(5.86,7.07)\end{array}$ & -1406.03 \\
\hline$(2 \mathrm{~d}) \mu=\mu_{0}+\mu_{\mathrm{w}}(t)+\mu_{1} \cos (2 \pi t), \sigma=\exp \left(\sigma_{0}+\sigma_{1} \cos (2 \pi t)\right)$ & $\begin{array}{c}5.91 \\
(5.48,6.47) \\
\end{array}$ & $\begin{array}{c}6.31 \\
(5.72,7.18) \\
\end{array}$ & $\begin{array}{c}6.67 \\
(5.92,7.86) \\
\end{array}$ & -1407.17 \\
\hline$(2 \mathrm{e}) \mu=\mu_{0}+\mu_{\mathrm{w}}(t)+\mu_{1} \cos (2 \pi t), \sigma=\exp \left(\sigma_{0}+\beta \mu_{\mathrm{w}}(t)\right)$ & $\begin{array}{c}5.97 \\
(5.58,6.46) \\
\end{array}$ & $\begin{array}{c}6.58 \\
(6.00,7.41)\end{array}$ & $\begin{array}{c}7.09 \\
(6.30,8.30)\end{array}$ & -1416.65 \\
\hline
\end{tabular}


From Table 1, it is evident that among the models of the first category and the ones of the second that include a scaling coefficient, $a$, in front of $\mu_{w}(t)$ in the location parameter, the one which incorporates a harmonic with a period of one year in both its location, $\mu$, and its scale, $\sigma$, parameters presents the lowest AIC value (model (1c) with $\mathrm{AIC}=-2251.12)$ and is therefore judged to be the best fitted model. The parameter vector of the selected model is $\boldsymbol{\theta}=\left(\mu_{0}, \mu_{1}, \mu_{2}, \sigma_{0}, \sigma_{1}, \sigma_{2}, \xi\right)$. The inclusion of more harmonics leads to an overparameterization of the extreme value model. For the models belonging to the second category, where the reconstructed part from the wavelet transform, $\mu_{w}(t)$ is included in the location parameter of the non-stationary point process without any scaling coefficient, the best fitted model for storm surges has a parameter vector $\boldsymbol{\theta}=\left(\mu_{0}, \mu_{l}, \sigma_{0}, \sigma_{l}, \xi\right)$, presenting an $\mathrm{AIC}=-$ 2233.41 (model (2d)). It should be noted that AIC values for the models which include the reconstructed part from the wavelet transform in their location parameter without a scaling coefficient and the rest of the models can not be performed (AIC values for the former models shown in italics).

Comparing the $95 \%$ confidence intervals resulting from models (1c) and (2d) of Table 1, it can be concluded that the latter, which incorporates the statistically significant features of the storm surge signal in its location parameter produces narrower confidence intervals for all return periods considered. More specifically, these differences reach up to almost $27 \%$, for return periods of 100 and 10000 years. Median return level estimates increase for the latter model up to almost 5\% for the return periods considered. But it should be noted that although the uncertainty seems to be reduced with model (2d), compared to the one that ignores the statistically significant periodicities of the signal (model (1c)), the largest observation of the sample is still underestimated. The largest observation of the sample which corresponds to a return period of 23 years, lies quite far from the rest of the extreme sample. Galiatsatou (2009) developed and used tests for outlier detection in extreme samples, to prove that there is very little evidence that this event should be excluded from the sample. The use of a different parameter estimation procedure, apart from the MLE, could provide better results.

From Table 2, among the representative models (1a)-(2b), the simplest one that provides a good fit to the data is model ( $1 \mathrm{~b})$ with $\mathrm{AIC}=-1416.20$. The selected model is represented by a parameter vector $\boldsymbol{\theta}=\left(\mu_{0}, \mu_{1}, \sigma_{0}, \sigma_{l}, \xi\right)$, including a single harmonic in its location and scale parameters. For the models belonging to the second category, where the reconstructed part from the wavelet transform, $\mu_{w}(t)$ is included in the location parameter of the non-stationary point process without any scaling coefficient, the best fitted model for wave heights has a parameter vector $\boldsymbol{\theta}=\left(\mu_{0}, \mu_{1}, \sigma_{0}, \beta, \xi\right)$, presenting an $\mathrm{AIC}=-$ 1416.65 (model (2e)). Although not comparable, the best fitted model with the component $\mu_{w}(t)$ results with an AIC value even lower than the one of the purely statistically evaluated model (1b). As for the storm surge data, comparison of the $95 \%$ confidence intervals for models (1b) and (2e) shows a significant reduction in uncertainty, when the latter one is implemented. Differences between the range of estimated intervals reach $25 \%, 19 \%$ and $20 \%$, for return periods of 100,1000 and 10000 years, respectively. Median return level estimates are slightly higher for model (2e).

The non-stationary model with parameters $\mu=\mu_{0}+\mu_{w}(t)+\mu_{l} \cos (2 \pi \mathrm{t}), \sigma=\exp \left(\sigma_{0}+\sigma_{l} \cos (2 \pi \mathrm{t})\right), \xi=$ $\xi_{0}$ for storm surge extremes and the one with parameters $\mu=\mu_{0}+\mu_{w}(t)+\mu_{l} \cos (2 \pi \mathrm{t}), \sigma=\exp \left(\sigma_{0}+\right.$ $\left.\beta \mu_{w}(t)\right), \xi=\xi_{0}$ for wave height extremes are graphically evaluated by means of the diagnostic plots for $Z$ and $W$ statistics, introduced earlier in the paper. Fig. 7(a,b) presents the diagnostic q-q (quantilequantile) plot for the $W$ statistic for storm surge and wave height extremes, respectively. Similar plots are also constructed for the $Z$ statistic, but are omitted here for the sake of brevity. From Fig. 7(b) there seems to be no reason to question the assumption of an exponential distribution with mean 1. From Fig. 7(a) it can be concluded that the selected model fits the extreme storm surge sample quite well, apart from the most extreme observation which is underestimated. However, still this model fits the extreme sample slightly better than the model with parameters $\mu=\mu_{0}+\mu_{1} \cos (2 \pi t)+\mu_{2} \sin (2 \pi t)$, $\sigma=\exp \left(\sigma_{0}+\sigma_{1} \cos (2 \pi t)+\sigma_{2} \sin (2 \pi t)\right)$ and $\xi=\xi_{0}(\operatorname{model} 1(\mathrm{c}))$.

In Tables 1 and 2 "aggregated" return level estimates are produced for both storm surge and wave height extremes, considering a representative year of the extreme data sample. But, as it was already mentioned within a non-stationary context it is better to think of the return level as a quantile of the distribution of the variable under study in the current year. Therefore "local" estimation of storm surge and wave height return levels for the selected models will also be performed using Eq. 13 to derive the maximum likelihood estimates and the delta method to estimate the associated $95 \%$ confidence intervals. Figures 8 and 9 present plots of instantaneous 95\% confidence intervals for 100 -years storm surge and wave height levels, respectively. Figure 8 compares models (1c) and (2d) of Table 1 for storm surge extremes, while Figure 9 compares models (1b) and (2e) of Table 2 for wave height extremes. 

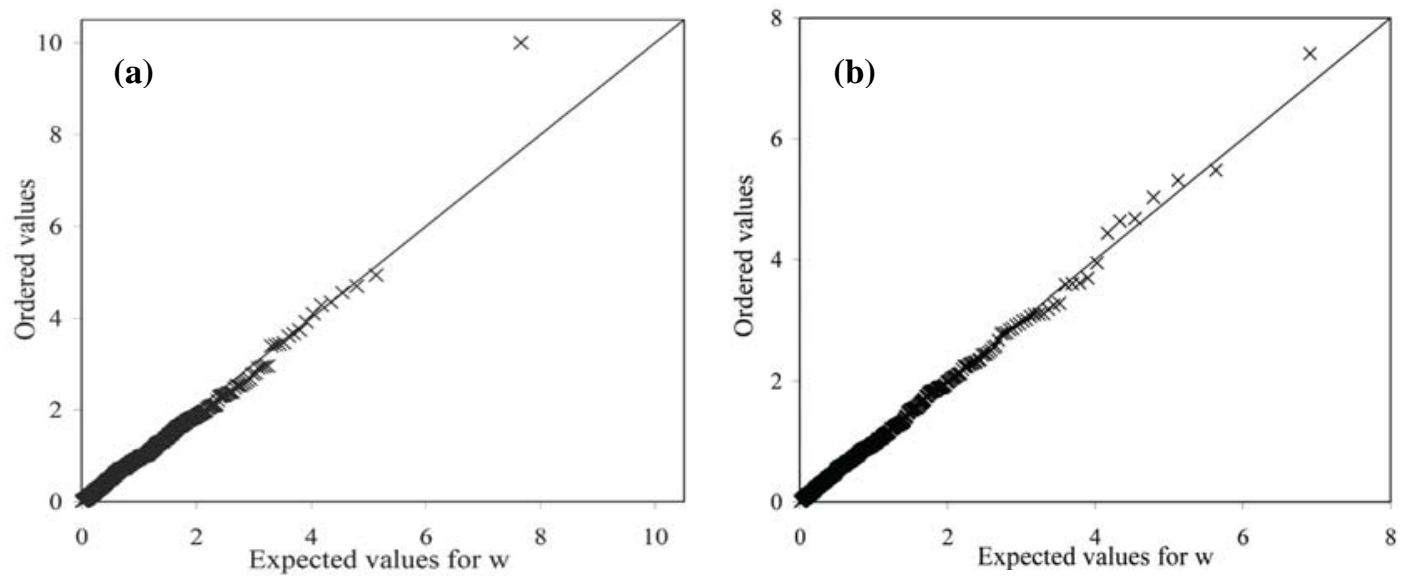

Figure 7. Quantile plots of $W$ statistics based on the selected model that incorporates the reconstructed part of the wavelet transform in its parameters for: a) storm surge extremes, b) wave height extremes at station Swb.

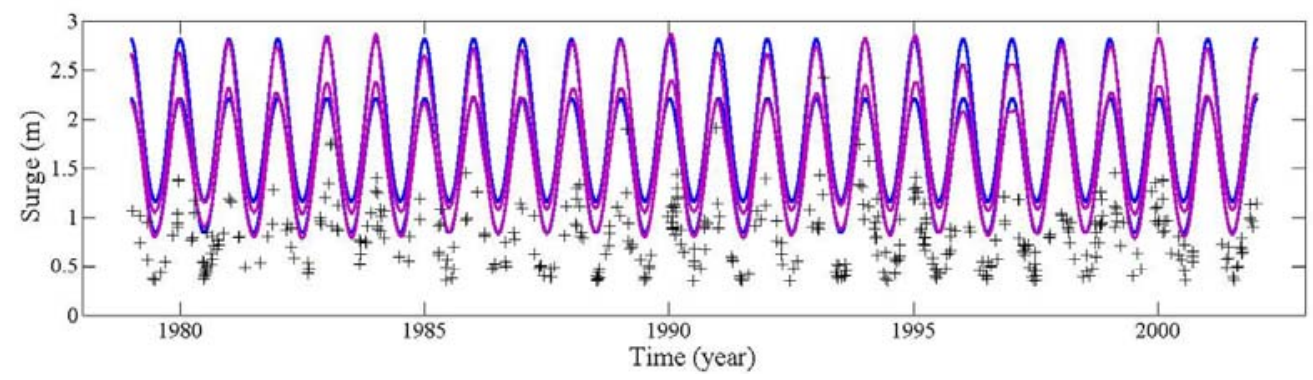

Figure 8. Estimated 95\% confidence intervals for storm surge data at station Swb for model (1c) -blue linesand model (2d) -purple lines- of Table 1.

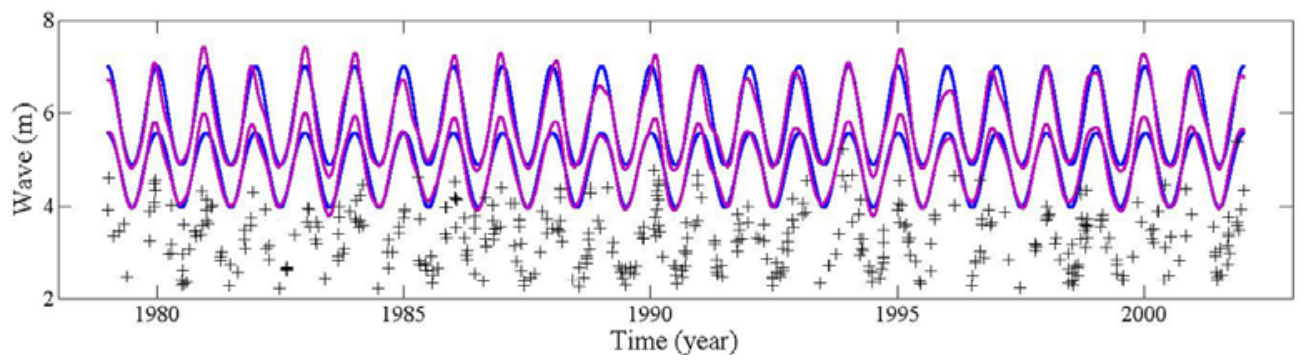

Figure 9. Estimated 95\% confidence intervals for wave height data at station Swb for model (1b) -blue linesand model (2e) -purple lines- of Table 2.

From Figures 8 and 9 it can be noticed that the model which introduces the component $\mu_{w}(t)$, estimated using the wavelet analysis, in the location parameter of the non-stationary point process (purple lines) produces narrower confidence intervals for both storm surges and wave heights. For storm surges and for a return period of 100 -years, the range of the $95 \%$ confidence interval is estimated narrower for the EV model which incorporates $\mu_{w}(t)$ up to $28 \%$, while for wave heights this proportion reaches $30 \%$. Thus, the selected model with the wavelet component reduces uncertainty in return level estimation compared to a simpler non-stationary model with harmonic functions estimated using the MLE procedure.

\section{CONCLUSIONS}

In the present work a non-stationary point-process model is combined with a signal processing technique, the wavelet transform, inquiring a possible reduction in uncertainty of extreme quantiles when prominent "periodicities" of the marine variables examined are included in the extremal analysis. 
The datasets used in the present work are storm surge and wave height observations in a selected location of the North Sea. The wavelet transform is first used to detect the significant "periodicities" of the signals by means of the wavelet global and scale-averaged power spectra and then it is utilized to reconstruct the part of the time series, represented by these statistically significant and prominent features. The reconstructed part of the series variability is incorporated in the location and/or in the scale parameter of the non-stationary point process model, together with selected harmonic functions and a time-varying threshold providing a uniform crossing rate within the year, formulating a number of candidate extreme value models. The quality of the fitted models is assessed by means of the AIC (Akaike Information Criterion), as well as by means of diagnostic quantile plots. The main conclusions of the research are summarized below:

1. The wavelet spectra for the Morlet and the Mexican hat wavelets reveal an obvious "periodicity", expressed with high wavelet power near the one-year band along a significant part of the storm surge and the wave time series. The one-year "periodicity" is evident from the global wavelet spectrum and seems to be statistically significant at a $5 \%$ significance level for both marine variables considered.

2. For the storm surge series statistically significant periodicities are revealed in higher periods possibly representing the inter-annual cycles of NAO.

3. The non-stationary model with parameters $\mu_{=} \mu_{0}+\mu_{w}(t)+\mu_{1} \cos (2 \pi t), \sigma=\exp \left(\sigma_{0}+\sigma_{1} \cos (2 \pi t)\right)$ and $\xi=$ $\xi_{0}$ is judged to represent the variability of the storm surge data sufficiently well compared to other models. It accounts for the main "periodicities" of the signal in a more natural way, without estimating the contribution of each one of them separately using statistical techniques.

4. The non-stationary model with parameters $\mu=\mu_{0}+\mu_{w}(t)+\mu_{1} \cos (2 \pi t), \sigma=\exp \left(\sigma_{0}+\beta \mu_{w}(t)\right)$ and $\xi=\xi_{0}$ is judged to represent the variability of the wave height extremes better than pure statistical periodical models.

5. The aforementioned models provide the narrowest range of $95 \%$ return level confidence intervals, representing a reduction in uncertainty in the estimation procedure.

6. For storm surges the $95 \%$ confidence intervals for "aggregated" return levels estimated using the non-stationary point process which includes $\mu_{w}(t)$ are narrower, compared to the ones estimated by the model which incorporates the same harmonics without the component from the inverse wavelet transform up to $27 \%$, for return periods of 100 and 10000 years.

7. For wave heights uncertainty in "aggregated" return level estimates is reduced using the nonstationary point process model that includes $\mu_{w}(t)$ up to $25 \%$, for the return periods considered.

8. When a local estimation of return levels is performed, uncertainty reductions in extreme predictions reach $28 \%$ and $30 \%$, for storm surges and wave heights, respectively, for a return period of 100 years.

9. Reducing uncertainty in wave height and storm surge return levels, using the proposed methodology, also reduces uncertainty in beach profile, flood extent and structural reliability estimation.

\section{ACKNOWLEDGMENTS}

The support of the European Commission through FP7.2009-1, Contract 244104 - THESEUS "Innovative technologies for safer European coasts in a changing climate", is gratefully acknowledged. The software used for the wavelet analysis was based on Wavelet software provided by C. Torrence and G. Compo through the URL: http://paos.colorado.edu/research/wavelets/.

\section{REFERENCES}

Butler, A. 2005. Statistical modelling of synthetic oceanographic extremes, Ph.D. Thesis, Lancaster University, London, UK.

Callaghan, D.P., P. Nielsen, A. Short R. and Ranasinghe. 2008. Statistical simulation of wave climate and extreme beach erosion, Coastal Engineering, 55(5), 375-390.

Coles, S. 2001. An introduction to statistical modelling of extreme values, Springer Series in Statistics

Coles, S. and J. Tawn. 1996. A Bayesian analysis of extreme rainfall data, Applied Statistics, 45(4), 463-478.

Coles, S. and J. Tawn. 2005a. Bayesian modeling of extreme surges on the UK east coast, Philosophical Transactions of the Royal Statistical Society A, 363, 1387-1406.

Coles, S. and J. Tawn. 2005b. Seasonal effects of extreme surges, Stochastic Environmental Research and Risk Assessment, 19, 417-427. 
Egozcue, J.J., V. Pawlowsky-Glahn and M.I. Ortego. 2005. Wave-Height Hazard Analysis in Eastern Coast of Spain: Bayesian Approach Using Generalized Pareto Distribution, Advances in Geosciences, 2, 25-30.

Farge, M. 1992. Wavelet transforms and their applications to turbulence, Annual Review of Fluid Mechanics, 24, 395-457, doi:10.1146/annurev.f1.24.010192.002143

FLOODsite: Integrated Flood Risk Analysis and Management Methodologies. 2004-2009, European Research Project, FP6, www.floodsite.net

Galiatsatou, P. 2009. Statistical estimation methods for extreme events simulation. Applications to storm surge, rainfall and wave data, $\mathrm{PhD}$ Dissertation, Division of Hydraulics and Environmental Engineering, Department of Civil Engineering, A.U.Th, Greece (in greek)

Galiatsatou, P. and P. Prinos. 2011, Modeling non-stationary extreme waves using a point process and wavelets, Stochastic Environmental Research and Risk Assessment, 25(2), 165-183.

Huerta, G. and B. Sanso. 2007. Time varying models for extreme values, Environmental and Ecological Statistics, 14, 285-299.

Kijewski-Correa, T. and A. Kareem. 2007. Performance of wavelet transform and empirical mode decomposition in extracting signals embedded in noise, Journal of Engineering Mechanics, ASCE, 133(7), 849-852

Liu, P.C. and A.V. Babanin. 2004. Using wavelet spectrum analysis to resolve breaking events in the wind wave time series, Annales Geophysicae, 22, 3335-3345.

Markovic, D. and M. Koch. 2005. Wavelet and scaling analysis of monthly precipitation extremes in Germany in the $20^{\text {th }}$ century: Interannual to interdecadal oscillations and the North Atlantic Oscillation influence, Water Resources Research, 41, W09420, doi: 10.1029/2004WR003843

Massel, S.R. 2001. Wavelet analysis for processing of ocean surface wave records, Ocean Engineering, 28, 957-987.

Méndez, F.J., M. Menéndez, A. Luceño and I.J. Losada. 2006 Estimation of the long-term variability of extreme significant wave height using a time-dependent Peak Over Threshold (POT) model. Journal of Geophysical Research, 11, C07024. doi:10.1029/2005JC003344.

Méndez, FJ, M. Menéndez, A. Luceño, R. Medina and N.E. Graham. 2008. Seasonality and duration in extreme value distributions of significant wave height, Ocean Engineering, 35, 131-138.

Menéndez, M., F.J., Méndez, C. Izaguirre, A. Luceño and I.J. Losada. 2009. The influence of seasonality on estimating return values of significant wave height, Coastal Engineering, 56, 211219.

Menéndez, M., F.J. Méndez, I.J. Losada and N.E. Graham. 2008. Variability of extreme wave heights in the northeast Pacific Ocean based on buoy measurements, Geophysical Research Letters, 35, L22607. doi: 10.1029/2008GL035394

Morgan, M.G. and M. Henrion. 1990. Uncertainty: a guide to dealing with uncertainty in quantitative risk and policy analysis, Cambridge University Press, New York.

Pandey, M.D., P.H.A.J.M. van Gelder and J.K. Vrijling. 2001. The estimation of extreme quantiles of wind velocity using L-Moments in the Peaks-Over-Threshold approach, Structural Safety, 23, 179-192.

Prinos, P., Th. Koftis, and P. Galiatsatou. 2010. Wavelet analysis of wave propagation over Posidonia Oceanica, Proceedings of Coastlab10 Conference, Barcelona, Spain (full paper in CD-ROM)

Sánchez-Arcilla, A., D. Gonzalez-Marco, N. Doorn and A. Kortenhaus. 2008. Extreme values for coastal, estuarine and riverine environments, Journal of Hydraulic Research, 46(2), 183-190.

Smith, R. L. 1989. Extreme value analysis of environmental time series: an example based on ozone data (with discussion), Statistical Science, 4, 367-393.

Torrence, Ch. and G. P. Compo. 1998. A practical guide to wavelet analysis, Bulletin of the American Meteorological Society, 79(1), 61-78.

Torrence, C. and P. Webster. 1999. Interdecadal changes in the ENSO-Moonsoon System, Journal of Climate, 12, 2679-2690.

van Gelder, P.H.A.J.M. 1999. Statistical methods for the risk-based design of civil structures, $\mathrm{PhD}$ Dissertation, The Netherlands

van Gelder, P.H.A.J.M. and C. Mai. 2008. Distribution functions of extreme sea waves and river discharges, Journal of Hydraulic Research, 46(2), 280-291.

Zachary, S., G. Feld, G. Ward and J. Wolfram. 1998. Multivariate extrapolation in the offshore environment, Applied Ocean Research, 20, 273-295, doi:10.1016/S0141-1187(98)00027-3. 